\title{
A phase II study of mitomycin-C and S-1 as third-line chemotherapy in patients with advanced colorectal cancer
}

\author{
JUNG HAN KIM ${ }^{1}$, HYEONG SU KIM ${ }^{1}$, DAE RO CHOI ${ }^{1}$, GEUNDOO JANG ${ }^{1}$, JUNG HYE KWON ${ }^{1}$, \\ HO YOUNG KIM ${ }^{1}$, JOO YOUNG JUNG ${ }^{1}$, HYO JUNG KIM ${ }^{1}$, HUN HO SONG ${ }^{1}$, YUN HO SHIN ${ }^{1}$, \\ SO YOUNG JUNG ${ }^{2}$, BYUNG CHUN KIM ${ }^{2}$ and DAE YOUNG ZANG ${ }^{1}$ \\ Departments of ${ }^{1}$ Internal Medicine and ${ }^{2}$ Surgery, Hallym Medical Center, \\ Hallym University College of Medicine, Seoul 150-950, Republic of Korea
}

Received April 20, 2011; Accepted July 19, 2011

DOI: 10.3892/ol.2011.367

\begin{abstract}
This study was conducted to evaluate the efficacy and safety of the combination of mitomycin-C (MMC) and S-1 as third-line chemotherapy for patients with advanced colorectal cancer (CRC) showing resistance to irinotecan- and oxaliplatin-containing regimens. Patients were recruited into the study from January 2009 and 10 patients were enrolled for 10 months. However, since no patients had shown a response by 10 months, the study was terminated early according to the protocol. MMC $7 \mathrm{mg} / \mathrm{m}^{2}$ was administered intravenously on day 1 every 6 weeks in the first 4 cycles. S- 1 was administered twice daily at $35 \mathrm{mg} / \mathrm{m}^{2}$, within $1 \mathrm{~h}$ of meals on days $1-14$. Following a rest for 7 days, S-1 was administered again on days 22-35, followed by a 7-day rest. A total of 14 cycles were delivered for 10 patients. All 10 patients were assessable for response. A total of 3 patients (30\%) had stable disease and the remaining 7 showed disease progression. With a median follow-up of 7 months, the median overall survival was 10.5 months. Grade 3-4 myelotoxicities included neutropenia in two patients, anemia in two and thrombocytopenia in one. Grade 1-2 nausea and vomiting developed in 5 patients. One patient experienced grade 3 diarrhea. Grade 1-2 hand-foot syndrome occurred in 4 patients. In conclusion, the combination of MMC and $\mathrm{S}-1$ as third-line chemotherapy in patients with advanced CRC appears to be well tolerated but has poor activity.
\end{abstract}

\section{Introduction}

Colorectal cancer (CRC) is the second most common cause of cancer-related mortality in Western countries. In South Korea, $\mathrm{CRC}$ is the fourth most commonly occurring malignancy, accounting for approximately $10 \%$ of newly diagnosed cancer

Correspondence to: Dr Hyeong Su Kim, Department of Internal Medicine, Kang Nam Sacred-Heart Hospital, Hallym University Medical Center, 948-1, Daelim-1 Dong, Youngdungpo-Gu, Seoul, 150-950, Republic of Korea

E-mail:nep2n@hallym.or.kr

Key words: colorectal cancer, mitomycin-C, S-1 cases (1). Approximately $30 \%$ of CRC patients present with advanced disease at first diagnosis, and $50 \%$ of patients who have received surgery eventually develop metastases during the course of their disease.

5-Fluorouracil (5-FU) combined with leucovorin (LV) had been widely used in the treatment of metastatic CRC, showing an overall response rate (RR) of $20-30 \%$. Since the 1990s, the introduction of irinotecan or oxaliplatin has extended the spectrum of therapeutic options. The addition of irinotecan or oxaliplatin to infused 5-FU and LV has shown a significant improvement in tumor response and patient survival (2-4). It is common practice to administer these two chemotherapeutic regimens sequentially for patients with metastatic CRC. However, no effective third-line chemotherapy exists currently for patients who are resistant to irinotecan or oxaliplatin combined with 5-FU/LV.

S-1 is an oral fluoropyrimidine, in which tegafur has been combined with gimeracil and potassium oxonate. S-1 has shown promising efficacy in untreated CRC. Moreover, in a phase II trial with metastatic CRC patients showing failure of irinotecan- and oxaliplatin-containing regimens, the overall RR obtained with S-1 monotherapy was $14.3 \%$ (5). Mitomycin-C (MMC) is an alkylating agent with an activity against adenocarcinoma of the stomach, pancreas, breast and colon. In a number of studies, MMC has shown a RR of $10-15 \%$ in advanced CRC $(6,7)$. MMC has a mild hematologic toxicity and is not associated with stomatitis or diarrhea. Since MMC has demonstrated synergistic activity with 5-FU (8), it is usually combined with 5-FU for clinical use. The MMC/5-FU combination was considered to be one of the most essential regimens for gastrointestinal types of cancer $(9,10)$; therefore, its role in combination with an oral fluoropyrimdine, such as $\mathrm{S}-1$, should be investigated.

This study was conducted to evaluate the efficacy and safety of the combination of MMC and S-1 as third-line chemotherapy for patients with advanced CRC showing resistance during treatment with irinotecan- and oxaliplatin-containing regimens.

\section{Materials and methods}

Study design. This trial was a phase II study of the MMC and S-1 combination in metastatic CRC patients showing 
resistance during treatment with irinotecan- and oxaliplatincontaining regimens. The primary end-points were overall RR and safety, and the secondary end-points were time to progression (TTP) and overall survival (OS).

According to Simon's two-stage optimal design, 29 patients were required for a statistical power of $80 \%$ and a false positive rate of $5 \%$, with a lower activity level of $10 \%$ and a higher activity level of $30 \%$. At the first stage, if none out of the initial 10 patients showed a response, the study was due to be terminated. Assuming a 10\% drop-out rate, 32 patients were planned to be enrolled for this study. The study was approved by the institutional review board, and all patients gave written informed consent prior to recruitment into the study.

Patients. Patients were recruited into the study from January 2009 and 10 patients were enrolled for 10 months. The patients included 7 males and 3 females, with a median age of 62 years (range 42-76). The eligibility criteria for the study were: histologically confirmed colorectal adenocarcinoma with bidimensionally measurable metastatic disease, age over 18 years, Eastern Cooperative Oncology Group (ECOG) performance status (PS) of $<2$, adequate bone marrow function (absolute neutrophil count $\geq 1.5 \times 10^{9} / 1$, platelet $\geq 100 \times 10^{9} / 1$ and hemoglobin $\geq 10 \mathrm{~g} / \mathrm{dl}$ ), adequate renal and hepatic function (serum creatinine $\leq 1.25 \mathrm{x}$ upper normal limit, hepatic enzymes and bilirubin $\leq 1.25 \mathrm{x}$ upper normal limit, prothrombin time $\leq 1.5 \times$ control) and documented disease progression during treatment with irinotecan- and oxaliplatin-containing regimens. Patients were ineligible if they had other malignancies, brain metastases or active infection.

Treatment schedule. MMC $7 \mathrm{mg} / \mathrm{m}^{2}$ was administered intravenously on day 1 every 6 weeks in the first 4 cycles. To prevent hemolytic uremic syndrome (HUS), MMC was restricted to a cumulative dose of $28 \mathrm{mg} / \mathrm{m}^{2}$. MMC dose was reduced by $25 \%$ if grade 3 or 4 hematologic toxicity occurred. MMC administration was terminated if there was hemolytic anemia, severe and prolonged thrombocytopenia or fragmented red cells on the peripheral blood smear.

The starting dose of S-1 was twice daily at $35 \mathrm{mg} / \mathrm{m}^{2}$. S-1 was administered within $1 \mathrm{~h}$ of meals on days 1-14. Following a rest for 7 days, S-1 was administered again on days 22-35, followed by a 7-day rest. S-1 dose was reduced by $10 \mathrm{mg} / \mathrm{m}^{2}$ a day if grade 3 or 4 hematologic or non-hematologic toxicity developed. Treatment courses were repeated every 6 weeks unless there was evidence of disease progression, unacceptable toxicity or the patient refused to continue treatment.

Response and toxicity evaluation. Baseline evaluation included physical examination, complete blood counts (CBC), peripheral blood smear, blood chemistries and radiological examinations. Physical examination, $\mathrm{CBC}$ and blood chemistry were performed every 3 weeks. Tumor assessment by CT scan was performed every 6 weeks. Patients were evaluated for response if they received more than one cycle of treatment. Response to therapy was assessed according to the guidelines of the Response Evaluation Criteria in Solid Tumors (RECIST) Committee. Toxicity was recorded according to the Common Toxicity Criteria of the National Cancer Institute (NCI-CTC, version 3.0). For toxicity analysis,
Table I. Patient characteristics.

No. of patients

\begin{tabular}{lc}
\hline Age, years & \\
Median & 62 \\
Range & $42-76$ \\
Gender & \\
Male & $7(70 \%)$ \\
Female & $3(30 \%)$ \\
Performance status (ECOG) & \\
$0-1$ & $7(70 \%)$ \\
2 & $3(30 \%)$ \\
Primary site & \\
Colon & $6(60 \%)$ \\
Rectum & $4(40 \%)$ \\
Site of metastases & \\
Liver & $5(50 \%)$ \\
Lymph node & $4(40 \%)$ \\
Lung & $3(30 \%)$ \\
Peritoneum & $2(20 \%)$ \\
Bone & $2(20 \%)$ \\
Histology & \\
Well, moderate & \\
Poor & $6(60 \%)$ \\
\hline
\end{tabular}

the worst data for each patient in all cycles of chemotherapy were used.

Statistical analysis. Response and toxicity data were analyzed using simple descriptive statistics. TTP was determined from the first day of chemotherapy until tumor progression or mortality. OS was calculated from the first day of treatment until the date of mortality. Survival curves were established by using the Kaplan-Meier method.

\section{Results}

Patient characteristics. According to the study design, at least one out of the 10 patients were required to show a response to continue the study. However, since no patients had shown a response by 10 months, the study was terminated early. Baseline characteristics of the enrolled patients are shown in Table I. A total of 7 patients (70\%) had an ECOG PS of 0 to 1 and 3 patients had an ECOG PS of 2. All 10 patients had multiple sites of metastases. The most common metastatic site was the liver, followed by the intra-abdominal lymph node, lung and peritoneum. A total of 4 patients had a poorly differentiated adenocarcinoma.

Treatment outcomes. A total of 14 cycles were delivered to the 10 patients. A total of 7 patients received 1 cycle and 2 patients received 2 cycles, and the remaining patient received 3 cycles. Chemotherapy was stopped due to disease progression in 9 patients and poor PS in one patient. A total of 9 patients were 


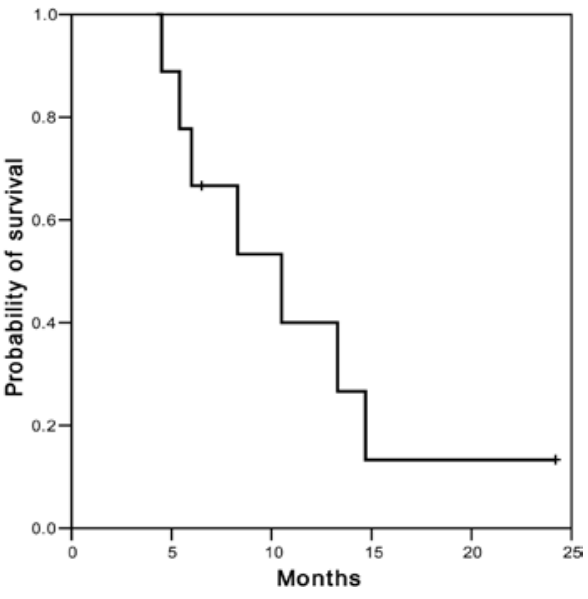

Figure 1. Overall survival.

not switched over to the following chemotherapy following disease progression. One patient received cetuximab plus irinotecan and experienced a partial response for 4 months.

All 10 patients were assessable for response. A total of 3 patients (30\%) had stable disease and the remaining seven showed disease progression. The duration of stable disease was 6 weeks in two patients and 3 months in one patient. With a median follow-up of 7 months, the median overall survival was 10.5 months (range 3.7-24.2). One patient remains alive with a follow-up period of 24.2 months.

Toxicity. The patients were evaluable for toxicity. The toxicity profile is shown in Table II. NCI-CTC grade 3-4 myelotoxicities were as follows: neutropenia in two patients, anemia in two patients and thrombocytopenia in one patient. No febrile neutropenia occurred. Grade 1-2 nausea and vomiting developed in five patients. One patient experienced grade 3 diarrhea. Grade 1-2 hand-foot syndrome occurred in four patients. No patients developed hemolytic anemia or HUS.

\section{Discussion}

In this phase II study, the efficacy of MMC plus S-1 was evaluated as third-line chemotherapy for patients with advanced CRC showing resistance to irinotecan- and oxaliplatin-containing regimens. This study was terminated early as none of the initial 10 patients showed a response. Although this regimen failed to show activity for these patients, we believe the results are worth reporting.

Irinotecan and oxaliplatin are widely used in combination with 5-FU/LV as first- and second-line treatment for CRC (2-4). Targeted compounds including cetuximab and bevacizumab may also be introduced into the treatment of advanced CRC $(11,12)$. However, few reports are currently available of salvage therapy for patients who are refractory to the first- and second-line chemotherapy $(5,13)$. Although cetuximab with or without irinotecan is a potential option for patients without k-ras mutation (14), its use is limited due to the high cost involved. Therefore, new chemotherapeutic regimens remain to be determined.

The rationale for combining MMC with fluoropyrimidine was based on the different cytotoxic mechanism of the drugs
Table II. Toxicity profile by grade.

\begin{tabular}{lcc}
\hline Toxicity & Grade 1-2(\%) & Grade 3-4 (\%) \\
\hline Hematologic toxicities & & \\
$\quad$ Neutropenia & $2(20)$ & $2(20)$ \\
Anemia & $3(30)$ & $2(20)$ \\
Thrombocytopenia & $2(20)$ & $1(10)$ \\
Non-hematologic toxicities & & \\
Nausea & $5(50)$ & $0(0)$ \\
Vomiting & $5(50)$ & $0(0)$ \\
Hand-foot syndrome & $4(40)$ & $0(0)$ \\
Diarrhea & $1(10)$ & $1(10)$ \\
Stomatitis & $3(30)$ & $0(0)$ \\
\hline
\end{tabular}

and their non-overlapping adverse effects. The combination chemotherapy of MMC and an oral fluoropyrimidine carbonate, capecitabine, has shown a synergistic effect in gastrointestinal tumors $(15,16)$. In the present study, we combined MMC and S-1 to improve antitumor activity. In a phase II study in patients with advanced gastric cancer, MMC plus $\mathrm{S}-1$ as second-line therapy has shown an objective RR of $21 \%$, with a tolerable toxicity profile (17).

None of the 10 patients who were enrolled at the first stage of this study showed a response. Therefore, the study was terminated early according to the protocol. Three patients showed stable disease, which was maintained for 1.5 to 3 months. Jeung et al have reported a phase II trial of S-1 monotherapy in metastatic CRC patients showing failure of irinotecan- and oxaliplatin-containing regimens (5). Of 26 patients, the overall RR was $14.3 \%$ and the disease control rate was $42.9 \%$. The difference in response between studies may be associated with a variety of patient characteristics and prognostic factors. The 10 enrolled patients in this study had shown disease progression during (not within 6 months after) treatment with irinotecan- and oxaliplatin-containing regimens. Four patients $(40 \%)$ had a poorly differentiated adenocarcinoma, compared to $10.7 \%$ in the study by Jeung et al. Therefore, these differences may have an impact on the tumor response in this study. Additionally, in a phase II trial of a combination of MMC and another oral fluoropyrimidine, capecitabine, as third-line chemotherapy in patients with advanced CRC, only one out of 19 patients (4.8\%) showed a partial response (13). These results suggest that the combination of MMC and an oral fluoropyrimidine, such as capecitabine or S-1, has poor activity in advanced CRC patients pretreated with irinotecan and oxaliplatin sequentially in combination with infused 5-FU/LV.

In terms of toxicity, the MMC and S-1 combination in this study showed favorable safety profiles. The most common toxicity was nausea and vomiting, followed by hand-foot syndrome. Hematologic toxicities were generally mild and manageable, and no febrile neutropenia developed. In association with MMC, none of the 10 patients developed hemolytic anemia or HUS.

In conclusion, the combination of $\mathrm{MMC}$ and $\mathrm{S}-1$ in advanced CRC patients pretreated with irinotecan- and 
oxaliplatin-containing regimens is well tolerated. However, the present result indicates that this regimen has poor activity for those patients. Therefore, attempts to develop other types of salvage therapy are required.

\section{References}

1. Bae JM, Won YJ, Jung KW and Park JG: Annual report of the Korean central cancer registry program 2000. Cancer Res Treat 34: 77-83, 2002.

2. Douillard JY, Cunningham D, Roth AD, et al: Irinotecan combined with fluorouracil compared with fluorouracil alone as first-line treatment for metastatic colorectal cancer: a multicentre randomised trial. Lancet 355: 1041-1047, 2000.

3. Saltz LB, Cox JV, Blanke C, et al: Irinotecan plus fluorouracil and leucovorin for metastatic colorectal cancer. Irinotecan Study Group. N Engl J Med 343: 905-914, 2000.

4. De Gramont A, Figer A, Seymour M, et al: Leucovorin and fluorouracil with or without oxaliplatin as first-line treatment in advanced colorectal cancer. J Clin Oncol 18:2938-2947, 2000.

5. Jeung HC, Rha SY, Cho BC, et al: A phase II trial of S-1 monotherapy in metastatic colorectal cancer after failure of irinotecan- and oxaliplatin-containing regimens. Br J Cancer 95: $1637-1641,2006$

6. Chester JD, Dent JT, Wilson G, Ride E and Seymour MT: Protracted infusional 5-fluorouracil (5-FU) with bolus mitomycin in 5-FU-resistant colorectal cancer. Ann Oncol 11: 235-237, 2000.

7. Seitz JF, Perrier H, Giovannini M, Capodano G, Bernardini D and Bardou VJ: 5-Fluorouracil, high-dose folinic acid and mitomycin $\mathrm{C}$ combination chemotherapy in previously treated patients with advanced colorectal carcinoma. J Chemother 10 258-265, 1998.

8. Russello O, Romanini A, Civalleri D, Rosso R, Nicolin A and Sobrero A: Time-dependent interactions between 5-fluorouracil and mitomycin $\mathrm{C}$ on a human colon carcinoma cell line, HCT-8, in vitro. Eur J Cancer Clin Oncol 25: 571-572, 1989.
9. Ross P, Norman A, Cunningham D, et al: A prospective randomized trial of protracted venous infusion 5-fluorouracil with or without motomycin $\mathrm{C}$ in advanced colorectal cancer. Ann Oncol 8: 995-1001, 1997.

10. Tebbutt NC, Norman A, Cunningham D, et al: A multicentre, randomized phase III trial comparing protracted venous infusion (PVI) 5-fluorouracil (5-FU) with PVI 5-FU plus mitomycin C in patients with inoperable oesophago-gastric cancer. Ann Oncol 13: 1568-1575, 2002.

11. Hurwitz H, Fehrenbacher L, Novotny W, et al: Bevacizumab plus irinotecan, fluorouracil, and leucovorin for metastatic colorectal cancer. N Engl J Med 350: 2335-2342, 2004.

12. Karapetis CS, Khambata-Ford S, Jonker DJ, et al: K-ras mutations and benefit from cetuximab in advanced colorectal cancer. N Engl J Med 359: 1757-1765, 2008.

13. Lim DH, Park YS, Park BB, et al: Mitomycin-C and capecitabine as third-line chemotherapy in patients with advanced colorectal cancer: a phase II study. Cancer Chemother Pharmacol 56: $10-14,2005$

14. Cunningham D, Humblet Y, Siena S, et al: Cetuximab monotherapy and cetuximab plus irinotecan in irinotecan-refractory metastatic colorectal cancer. N Engl J Med 351: 337-345, 2004.

15. Kikuyama S, Inada T, Oyama R and Ogata Y: Phase II study of mitomycin $\mathrm{C}$, cisplatin and 5-fluorouracil for advanced and recurrent gastric cancer. Anticancer Res 22: 3633-3636, 2002.

16. Kornek GV, Schuell B, Laengle F, et al: Mitomycin C in combination with capecitabine or biweekly high-dose gemcitabine in patients with advanced biliary tract cancer: a randomised phase II trial. Ann Oncol 15: 478-483, 2004

17. Park SH, Kim YS, Hong J, et al: Mitomycin C plus S-1 as second-line therapy in patients with advanced gastric cancer: a noncomparative phase II study. Anticancer Drugs 19: 303-307, 2008. 